\title{
The assessment of intermediate coronary lesions using intracoronary imaging
}

\author{
Jason Nogic ${ }^{1,2}$, Hamish Prosser ${ }^{2}$, Joseph O'Brien ${ }^{2}$, Udit Thakur ${ }^{1}$, Kean Soon ${ }^{2}$, George Proimos ${ }^{2}$, \\ Adam J. Brown ${ }^{1}$
}

${ }^{1}$ Monash Cardiovascular Research Centre, Monash University and MonashHeart, Monash Health, Melbourne, Victoria, Australia; ${ }^{2}$ Department of Cardiology, Eastern Health, Melbourne, Victoria, Australia

Contributions: (I) Conception and design: AJ Brown, J Nogic; (II) Administrative support: None; (III) Provision of study materials or patients: None; (IV) Collection and assembly of data: None; (V) Data analysis and interpretation: None; (VI) Manuscript writing: All authors; (VII) Final approval of manuscript: All authors.

Correspondence to: Dr. Adam J. Brown. Monash Cardiovascular Research Centre, Monash University and MonashHeart, Monash Health, 246 Clayton Road, Clayton, Melbourne, Victoria, Australia. Email: ajdbrown@me.com.

\begin{abstract}
Intermediate coronary artery stenosis, defined as visual angiographic stenosis severity of between $30-70 \%$, is present in up to one quarter of patients undergoing coronary angiography. Patients with this particular lesion subset represent a distinct clinical challenge, with operators often uncertain on the need for revascularization. Although international guidelines appropriately recommend physiological pressure-based assessment of these lesions utilizing either fractional flow reserve (FFR) or quantitative flow ratio (QFR), there are specific clinical scenarios and lesion subsets where the use of such indices may not be reliable. Intravascular imaging, mainly utilizing intravascular ultrasound (IVUS) and optical coherence tomography (OCT) represents an alternate and at times complementary diagnostic modality for the evaluation of intermediate coronary stenoses. Studies have attempted to validate these specific imaging measures with physiological markers of lesion-specific ischaemia with varied results. Intravascular imaging however also provides additional benefits that include portrayal of plaque morphology, guidance on stent implantation and sizing and may portend improved clinical outcomes. Looking forward, research in computational fluid dynamics now seeks to integrate both lesion-based physiology and anatomical assessment using intravascular imaging. This review will discuss the rationale and indications for the use of intravascular imaging assessment of intermediate lesions, while highlighting the current limitations and benefits to this approach.
\end{abstract}

Keywords: Optical coherence tomography (OCT); intravascular ultrasound (IVUS); coronary artery disease $(\mathrm{CAD})$

Submitted Feb 15, 2020. Accepted for publication Jun 23, 2020.

doi: $10.21037 / \mathrm{cdt}-20-226$

View this article at: http://dx.doi.org/10.21037/cdt-20-226

\section{Introduction}

Coronary artery disease (CAD) remains a major cause of morbidity and mortality worldwide. Many patients with obstructive CAD will experience symptoms of myocardial ischaemia and a reliable assessment of stenosis severity is required to ensure clinicians make appropriate revascularisation decisions. Traditionally, invasive coronary angiography has been the gold standard investigation to assess severity and extent of CAD. However, angiography is limited as it is a two-dimensional representation of a more complex three-dimensional structure. Interpretation of images can also be hampered by vessel foreshortening or overlay, along with challenges in reliably assessing stenosis severity in highly eccentric lesions. The fallibility of stenosis severity on angiography has been documented in previous studies, which demonstrate an inter-observer variance in diameter stenosis of $15-45 \%$. Thus, visual estimation 
of stenosis severity lacks precision with up to $30 \%$ of angiographic assessments found to be erroneous (1). While some of this subjectivity can be addressed through use of quantitative coronary angiography (QCA) software, there remains issues with spatial and temporal resolution along with an inability to account for extraluminal abnormalities, marked vessel tortuosity and lesion asymmetry (2). Three dimensional QCA has gone some way in addressing these concerns allowing fusion of two or more angiographic views. However, even 3D QCA cannot overcome the limitation of angiographic resolution (3).

Given these inherent difficulties in accurate stenosis assessment on angiography, patients often fall into a category termed 'intermediate' stenosis severity. Intermediate stenosis is defined as an angiographic stenosis of between $30-70 \%$ and presents a distinct clinical challenge with uncertainty in regards to optimal assessment and management strategy (4). Although its prevalence in the general population is not well known, registry data suggests it may be present in up to $25 \%$ of patients undergoing coronary angiography (5).

Invasive physiological assessment is recognized as the optimal method for assessment of intermediate angiographic lesions. Myocardial fractional flow reserve (FFR) is defined as the ratio between maximum coronary blood flow in a stenotic artery compared with maximum blood flow in the theoretical absence of stenosis. This assessment is undertaken during the conditions of maximal hyperaemia, to ensure the effects of the coronary microcirculation are minimised (6). Pressure derived FFR is the most commonly utilised invasive tool and has been found to be superior to angiography when used to guide revascularization strategy $(7,8)$. Recent data also now support the role of hyperaemicfree pressure derived indices, such as the instantaneous wave-free ratio (iFR) $(9,10)$. Both physiological approaches are supported by current European Society of Cardiology (ESC) guidelines on myocardial revascularization suggesting that, when evidence of ischaemia is not readily available, FFR or iFR should be utilized to assess the haemodynamic significance of intermediate grade coronary stenosis (11).

More recently, there has also been the development of imaging based technology that provides lesion-specific physiological measurements without the need for guidewirebased technology. The most well investigated method, termed Quantitative Flow Ratio (QFR), has demonstrated good diagnostic accuracy when compared to invasive indices. QFR utilises an image-based virtual FFR index, computed by three-dimesional QCA combined with coronary flow velocity estimated by thrombolysis in myocardial infarction (TIMI) frame count (12). In a series of 265 patients assessing diagnostic performance of QFR, there was strong correlation with both FFR $(r=0.863, \mathrm{P}<0.001)$ and $\mathrm{iFR}$ $(\mathrm{r}=0.740, \mathrm{P}<0.001)$ producing a diagnostic accuracy of $90.8 \%$ and $81.3 \%(\mathrm{P}<0.001)$, respectively (13). Despite these initial encouraging results, the uptake of QFR remains limited due to time constraints of calculating results in real-time, along with cost issues relating to software and licensing.

However, pressure wire assessment of intermediate lesions is not without its limitations and/or contraindications. Certain patient and lesion specific factors may decrease the efficacy of FFR and/or iFR and therefore physiological assessment may not be suitable in all patients. Patient specific characteristics may include; patient contraindication to adenosine (e.g., profound bradycardia), concurrent or recent ischaemia, significant left ventricular hypertrophy, severe aortic valve stenosis and situations where maximal hyperaemia (in the case of FFR) cannot be achieved due to significant microvascular impairment. In these scenarios, FFR may over- or underestimate lesion-specific ischaemia and may not be as reliable in guiding revascularization (14). Lesion specific features may also hamper physiological assessment including aorto-ostial, left main and tandem lesions along with segments of diffuse disease (15). These specific patient/lesion subsets may benefit from assessment with the use of intravascular imaging, chiefly intravascular ultrasound (IVUS) or optical coherence tomography (OCT). This review will therefore review the evidence and discuss the role of intravascular imaging in the assessment of intermediate coronary artery lesions.

\section{Basic principles of IVUS}

Intravascular ultrasound makes use of a miniaturized piezoelectric transducer mounted to a catheter tip to produce ultrasound signals, enabling quantitative assessment of the vessel wall and intimal atherosclerotic lesions. IVUS is now widely utilised as an adjunct to coronary angiography, allowing clinicians to better identify and characterize plaque morphology, measure reference luminal dimensions and optimize stent size, placement and deployment $(16,17)$. Several manufacturers provide IVUS systems globally, enabling the majority of catheterization laboratories to have reliable access to the technology. The capabilities of IVUS continue to evolve, with improving resolution by higher acoustic frequency (up to $60 \mathrm{MHz}$ for the opticross HD) and increasing pullback speeds; providing more accurate 
and rapid lesion assessment. Several post-processing mathematical methods have also been developed to improve characterization of plaque and tissue morphology above that of standard grey-scale IVUS imaging, including iMAP-IVUS, virtual-histology (VH-IVUS) and integrated-backscatter (IBIVUS) $(18,19)$. Further innovations have included integration of other intravascular imaging technologies such as infrared, fluorescent spectroscopy, or photoacoustic imaging with IVUS to better assess lesions for markers of vulnerability such as fibrous cap thickness and plaque composition (20). These multi-modal catheters are currently limited in their commercial availability, as they are yet to be proven to provide significant additional clinical benefit to patient management beyond grayscale IVUS alone.

\section{Clinical outcomes of IVUS-guided PCI}

Several large meta-analyses investigating the clinical benefits of IVUS-guided PCI with bare metal- (BMS) and drug eluting stents (DES) have been reported, with general consensus that IVUS improves patient outcomes $(21,22)$. Focusing on DES, meta-analyses of RCTs and observational studies investigating IVUS-guided PCI have reported significant reductions in all-cause and cardiac death, target lesion revascularization (TLR), myocardial infarction (MI), and stent thrombosis (ST) $(21,23,24)$; which has been confirmed at patient-level analysis (25). In complex lesions, IVUS reduced MACE and TLR with no effect on death or ST (26). These analyses however vary in their inclusion/exclusion criteria, with some limited to RCTs and others including observational studies, increasing the risk of confounding factors and treatment-selection bias. This was highlighted by Buccheri et al. who found that when their analysis was limited to only RCTs, IVUS-associated reduction in all-cause death was lost (23). Nevertheless, there is increasing consensus that IVUS-guided PCI improves clinical outcomes and now features in the American College of Cardiology (ACC) and ESC guidelines for guiding treatment of unprotected left-main coronary artery (LMCA) lesions (Class IIa, level B evidence), and is indicated for non-LMCA lesions of angiographically intermediate stenosis by the ACC guidelines (Class IIb, level B evidence) $(27,28)$.

\section{IVUS assessment of LMCA disease}

LMCA disease is particularly challenging to accurately assess on angiography, as the arterial segment is often short, calcified and has disease that frequently involves the bifurcation. Unsurprisingly, it is known that there is high interobserver variability and low (41\% to $59 \%)$ agreement when assessing angiographic lesion severity at this particular location $(29,30)$. Given the critical importance of the LMCA to the coronary circulation and consequently its high prognostic value, accurate lesion assessment is crucial in guiding clinical management.

IVUS has been widely used in the assessment of intermediate LMCA lesions for many years. Indeed, IVUSderived minimal lumen dimension is known to be a strong predictor of MACE (31). Numerous studies have sought to identify a standardized IVUS-derived measure that can be used to assist in guiding therapy, with the minimal lumen area (MLA) receiving the greatest interest (Figure 1). A wide range of MLA cut-off values have been proposed (between 4.5 and $7.5 \mathrm{~mm}^{2}$ ) to guide revascularisation versus medical management, based on the ability to correlate significantly with an FFR of $<0.8(32-36)$. The MLA cut-off of $6 \mathrm{~mm}^{2}$ is often used as the standard to defer revascularisation, as this value was validated in the largest prospective multicentre (LITRO) study to date of 354 patients from 22 centres across Spain. Utilising IVUS, de la Torre Hernandez et al. found no significant difference in either cardiac-death $(94.5 \%$ vs. $97.7 \%, \mathrm{P}=0.5)$ or eventfree survival $(80.6 \%$ vs. $87.3 \%, \mathrm{P}=0.3)$ between those who underwent revascularisation (MLA $<6 \mathrm{~mm}^{2}, \mathrm{n}=179$ ) or in those is which it was deferred (MLA $\geq 6 \mathrm{~mm}^{2}, \mathrm{n}=186$ ) after 2 years (34). Of the 179 deferred patients, just 8 required revascularisation and this was predominantly due to stable angina, with no significant clinical, angiographic or ultrasonographic differences from those not requiring revascularisation. This MLA cut-off is supported by a prior study that identified an MLA cut-off of $5.9 \mathrm{~mm}^{2}$ correlated significantly with FFR $<0.75$ in 55 patients with moderate LMCA stenosis, with $93 \%$ sensitivity and $94 \%$ specificity (Table 1) (32). Opinion however does vary. The Mayo Clinic (USA) suggested a higher MLA cut-off value of $7.5 \mathrm{~mm}^{2}$, derived from the mean -2 standard deviations of MLA from a cohort of 121 patients with angiographically normal/minimally diseased LMCA (33). Subsequent longterm follow-up of 214 patients with intermediate LMCA lesions at $3.3 \pm 2.0$ years revealed no significant difference in MACE (TVR, MI, death) between those that underwent revascularization $(\mathrm{n}=83)$ or were deferred $(\mathrm{n}=131)$ using an MLA cut-off of $7.5 \mathrm{~mm}^{2}$ alone (33).

Smaller MLA cut-off values have been suggested based on studies from South Korea, in which $4.5-4.8 \mathrm{~mm}^{2}$ 


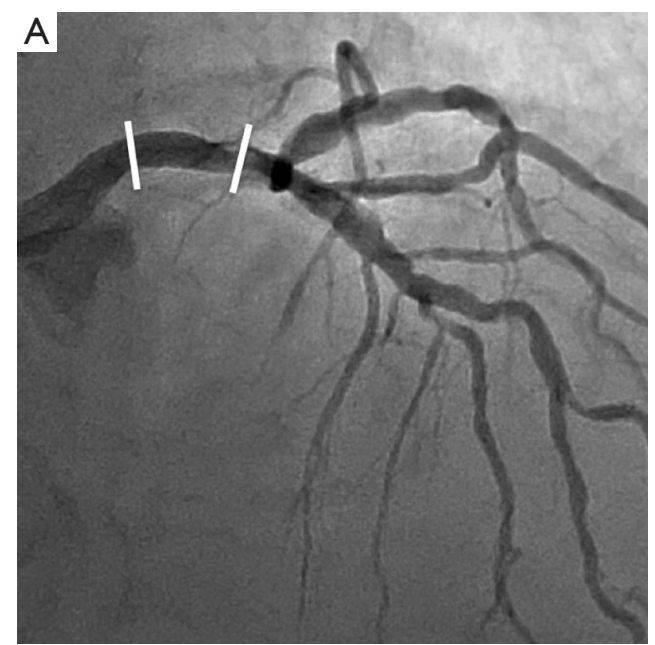

B
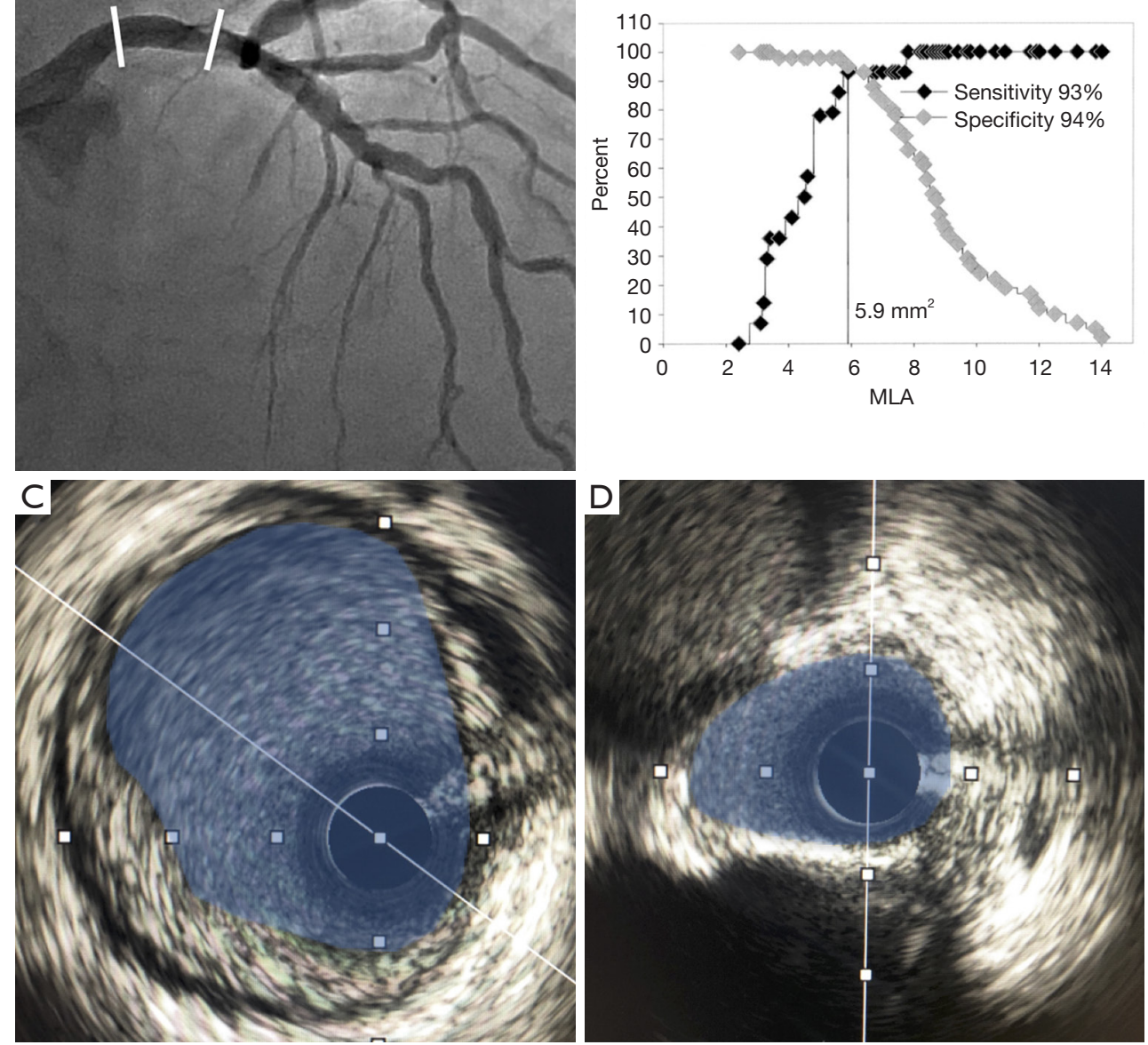

Figure 1 Intravascular ultrasound assessment of an intermediate left main coronary artery stenosis. Eccentric and calcified distal left main coronary artery (LMCA) lesion (A). Representative cross-sectional intravascular ultrasound (IVUS) images of the proximal reference segment with highlighted minimal luminal area (MLA) (highlighted blue) (C) and target lesion MLA (blue) highlighting eccentricity and superficial calcification (D). The calculated MLA (shaded blue) was $5.1 \mathrm{~mm}^{2}$, which is below the safe deferral threshold of 6 mm (B). Panel B reproduced from Jasti et al. Circulation, 2004 (32).

correlated significantly with FFR $<0.8$ in two studies, and an MLA of $4.1 \mathrm{~mm}^{2}$ correlated more significantly with FFR $<0.75$ (Table 1) $(35,36)$. Moreover, the same study found that among 40 lesions with MLA $<6.0 \mathrm{~mm}^{2}, 38 \%$ had FFR $\geq 0.8$, and $58 \%$ had FFR $\geq 0.75$ (35). These studies suggest that ethnicity and possibly body habitus should be taken into consideration in determining a safe MLA cutoff value in clinical practice. However, when taken together, these studies highlight that an IVUS-defined MLA cutoff of $>6 \mathrm{~mm}^{2}$ appears to be a safe deferral threshold for revascularisation across Western and Asian populations.

IVUS guided LMCA PCI has been found to significantly reduce all-cause mortality by $40 \%$, cardiac death by $53 \%$, plus reduce the risk of stent thrombosis and TLR in a meta-analysis of 10 studies with 6,480 patients undergoing IVUS-versus angiographic-guided stent insertion alone (51). Sub-group analysis revealed this significance was maintained across different study types, ethnic populations, and duration of follow-up (both $<3$ and $\geq 3$ years). A more recent meta-analysis of 7 studies including 4,592 patients also found IVUS guidance to significantly reduce allcause death, cardiac death, stent thrombosis and MI, but had no significant benefit on TLR, likely due to the study being underpowered (52). It must be noted that only one 
Table 1 Optimal IVUS-defined minimal luminal area (MLA) values to best predict FF

\begin{tabular}{|c|c|c|c|c|c|c|c|}
\hline Study & Patient/lesions & FFR & Best MLA cut-off $\left(\mathrm{mm}^{2}\right)$ & Sensitivity (\%) & Specificity (\%) & NPV (\%) & PPV (\%) \\
\hline Park 2014 (36) & $112 / 112$ & 0.80 & 4.5 & 78.7 & 80.4 & 75.9 & 82.8 \\
\hline Jasti 2004 (32) & $55 / 55$ & 0.75 & 5.9 & 93.0 & 95.0 & - & - \\
\hline Kang 2011 (35) & $55 / 55$ & $<0.80$ & 4.8 & 89.0 & 83.0 & 89.0 & 82.0 \\
\hline \multicolumn{8}{|l|}{ Non-LMCA studies } \\
\hline Takagi 1999 (37) & $42 / 51$ & 0.75 & 3.00 & 83.0 & 92.3 & - & - \\
\hline Lee 2010 (38) & $94 / 94$ & 0.75 & 2.00 & 87.9 & 78.9 & - & - \\
\hline Briguori 2001 (39) & $43 / 53$ & 0.75 & 4.00 & 92.0 & 56.0 & 96.0 & 46.0 \\
\hline \multirow{2}{*}{ Koo 2011 (40) } & & & Mid-LAD 2.75 & 73.0 & 78.0 & 83.0 & 68.0 \\
\hline & & & RCA 3.00 & 86.0 & 50.0 & 96.0 & 22.0 \\
\hline Ben-Dor 2012 (41) & $185 / 205$ & 0.80 & 3.09 & 69.2 & 79.5 & - & - \\
\hline Kang 2012 (42) & $692 / 784$ & 0.80 & 2.40 & 84.0 & 63.0 & 90.0 & 48.0 \\
\hline \multirow[t]{2}{*}{ Yang 2014 (43) } & $206 / 206$ & 0.80 & Prox-LAD 3.20 & 85.1 & 66.7 & - & - \\
\hline & & & Mid-LAD 2.50 & 65.1 & 87.7 & - & - \\
\hline \multirow[t]{2}{*}{ Han 2014 (44) } & $822 / 881$ & 0.80 & 2.75 & 61.0 & 63.0 & 73.0 & 49.0 \\
\hline & $580 / 623$ & 0.80 & 2.75 & 65.0 & 68.0 & - & - \\
\hline \multirow[t]{2}{*}{ Ben-Dor 2011(49) } & $84 / 92$ & 0.80 & 3.20 & 69.2 & 68.3 & - & - \\
\hline & & 0.75 & 2.80 & 79.7 & 80.3 & - & - \\
\hline Brown 2017 (50) & $89 / 92$ & 0.80 & 2.65 & 58.3 & 67.6 & - & - \\
\hline
\end{tabular}

NPV, negative predictive value; PPV, positive predictive value; MLA, minimal luminal area; FFR, fractional flow reserve; LMCA, left main coronary artery.

randomized controlled trial was included in each of these meta-analyses and, to our knowledge, just one further prospective RCT has been published since, in which IVUSguidance significantly reduced MACE (driven by reduced cardiac death), with no significant influence on MI, TLR or ST in 336 patients (53). How IVUS-guidance reduced mortality while having no significant effect on MI, ST or TLR remains to be elucidated. In the aforementioned trial the authors suggest it is likely related to reduced procedural adverse events, whereby IVUS enables greater characterisation of the vessel and lesion, improving stent sizing, placement, expansion and apposition, resulting in greater mean stent diameter (53). This is supported by a patient-level pooled registry analysis where IVUS improved clinical outcomes post-PCI in LMCA lesions (54).

LMCA lesions are frequently associated with downstream 

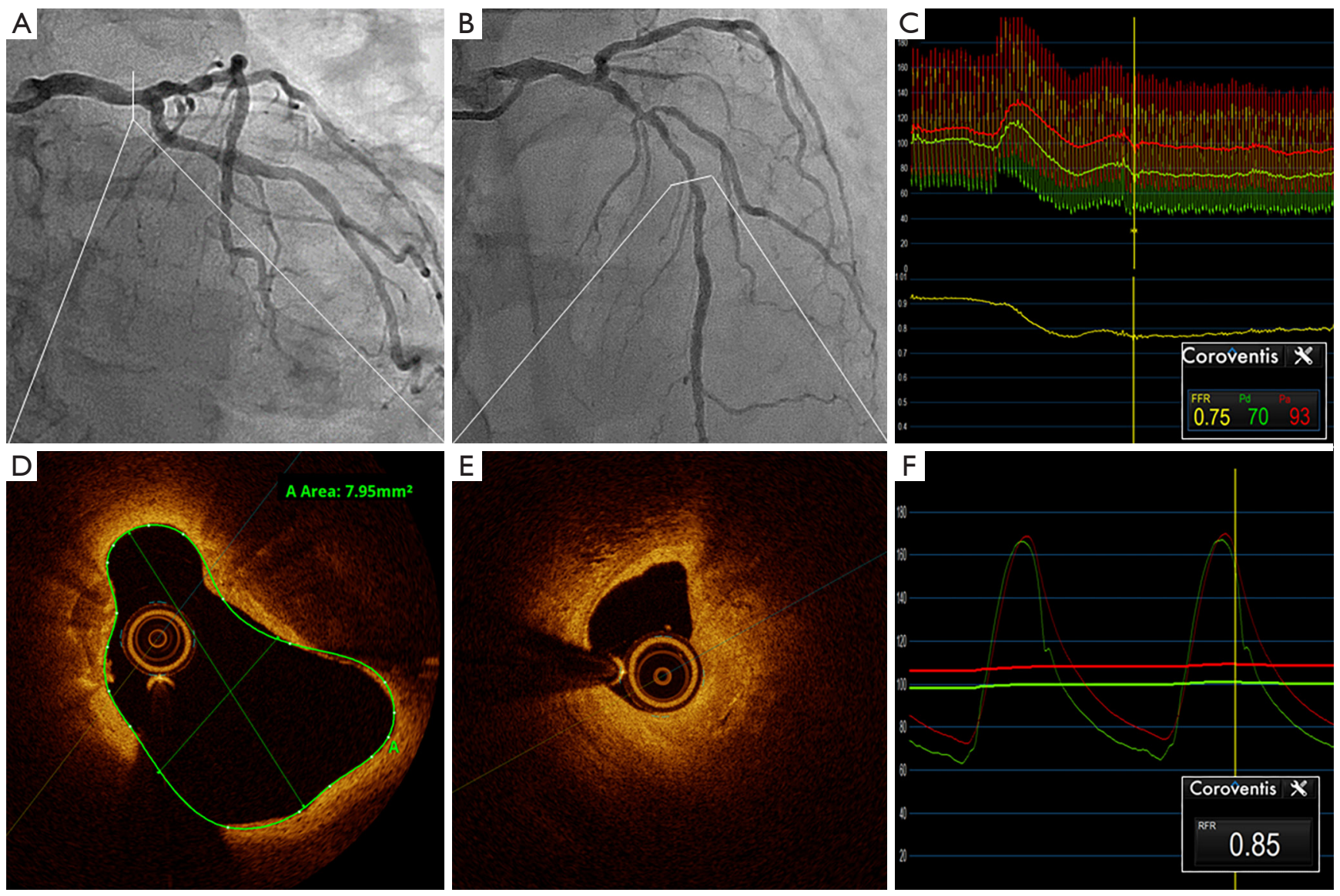

Figure 2 Hybrid use of intracoronary imaging and physiology to guide coronary revascularisation. Coronary angiography demonstrating intermediate distal left main coronary artery (LMCA) stenosis (A) with downstream severe disease in the mid left anterior descending (LAD) artery (B). Both fractional flow reserve (C) and resting full-cycle ratio (F) assessment confirm ischaemia within the LAD territory. Optical coherence tomography imaging demonstrates the minimal luminal area within the LMCA is $7.95 \mathrm{~mm}^{2}$ (D), highlighting that targeted revascularisation of the proximal to mid LAD should be considered and precluding need for intervention of the LMCA.

and/or multivessel disease (55), potentially confounding the ability of FFR to accurately assess the true functional significance of the LMCA stenoses. The effect of downstream disease was assessed by Fearon $e t$ al., who demonstrated that true FFR was significantly lower than that measured in the presence of a stenosis $(0.81 \pm 0.08 v s .0 .83 \pm 0.08, \mathrm{P}<0.001)$, even when the transducer was placed in a non-diseased vessel (56). Although the magnitude of this discrepancy is small and unlikely to affect clinical decision making in the vast majority of cases, the authors did note that the functional significance of LMCA disease cannot be accurately determined in cases where FFR in the non-diseased vessel is between 0.81 and 0.85 and in the presence of deep ischaemia due to severe downstream stenosis $(\mathrm{FFR}<0.45)$. These scenarios raise the possibility that intravascular imaging may be of use in assisting clinicians make revascularisation decisions during angiography (Figure 2).

This diagnostic value of IVUS in LMCA lesions is recognised in ESC guidelines, recommending IVUS should be considered to assess the severity of unprotected left main lesions (Class IIa Level B) and considered to optimize treatment of unprotected left main lesions (Class IIa Level B). However, a MLA cut-off value is not yet detailed in guidelines, likely due to the discrepancies across reports and lack of large trial data (28).

\section{IVUS assessment of non-LMCA lesions}

The current gold standard to assess significance of nonLMCA intermediate lesions remains physiological 
assessment (57). However, given the relative ease and safety of IVUS, several studies have sought to identify IVUSderived criteria (namely MLA) that can identify significant lesions with high diagnostic accuracy. To date, studies have generally reported poor diagnostic accuracy (66-74\%) of IVUS-derived MLA in correlating with FFR $<0.8$ in nonLMCA intermediate lesions (50). One promising study has reported an $83 \%$ sensitivity and $92.3 \%$ specificity to identify a FFR $\leq 0.75$ (MLA cut-off $3 \mathrm{~mm}^{2}$ ) (37); however, subsequent studies have generally reported poorer diagnostic capability (Table 1$)(42,46,58)$, and in one study of small vessels $(<2.8 \mathrm{~mm}$ wide and $<20 \mathrm{~mm}$ long) no correlation was found (59). Recent meta-analyses of non-LMCA studies report mean MLA cut-off of $\sim 2.60 \mathrm{~mm}^{2}$ with sensitivity and specificity of $75-79 \%$ and $65-66 \%$ respectively, to identify a FFR $\leq 0.8(60,61)$. Further diagnostic parameters are outlined in Table 1, in which IVUS-derived MLA is associated with (generally) far greater negative predictive values than positive predictive values, indicating that IVUS-derived MLA can provide clinical benefit in identifying stenoses acceptable for deferral, but should not be used in isolation to validate revascularisation of non-LMCA vessels.

The diagnostic accuracy of IVUS is further driven by vessel location and anatomical variants. For example, a significant correlation between MLA and FFR $<0.8$ was identified in major epicardial vessels $>3.5 \mathrm{~mm}$, but not side branches $<2.25 \mathrm{~mm}$ in 77 patients with intermediate ostial lesions (62). Table 1 lists the range of MLA cut-off values $\left(2-4 \mathrm{~mm}^{2}\right)$ reported in non-LMCA intermediate lesions including results from Koo et al., that revealed the need for different cut-off MLA values based on lesion location, and suggests that IVUS is unsuitable in vessels with high anatomical variation (40). Altering MLA cut-off values based on vessel diameter has been supported in one RCT in which 400 pairs of propensity score matched patients underwent PCI with a MLA of $<4 \mathrm{~mm}^{2}$ in vessels $>3 \mathrm{~mm}$, and $<3.5 \mathrm{~mm}^{2}$ in vessels $2.5-3 \mathrm{~mm}$, or FFR $\leq 0.75$ (63). MACE-free survival was equivalent between FFR and MLA-guided groups up to 2 years follow-up, with significantly more interventions performed in the IVUS compared with FFR group $(48.8 \%$ vs. $28 \% \mathrm{P}<0.001)$. Similarly, Nam et al. found IVUS-guidance (MLA cutoff $<4 \mathrm{~mm}^{2}$ ) resulted in a greater number of interventions compared to FFR-guidance $(\leq 0.8)$ in 167 patients with intermediate coronary lesions, with no significant difference in MACE at 1 year. They also calculated that reducing the MLA cut-off to $<3 \mathrm{~mm}^{2}$ equalized the number of interventions between groups, concluding that both FFR and IVUS were suitable for use in guiding PCI in patients with intermediate lesions (64). Increased stent use in IVUSguidance is often reported, with Park et al. also finding IVUS-guided therapy is associated with a greater number (as well as larger and longer stents used) with no improvement in patient outcomes, but rather a significant increase in target lesion failure predominantly due to periprocedural MI in a non-randomized post-hoc analysis of 463 matched patients from the EXCELLENT trial (65).

IVUS-derived MLA has also been shown to correlate significantly with physiological indices other than FFR, including iFR, diastolic pressure ratio (dPR), and resting full-cycle ratio (RFR) (66). Despite the significant correlation, the diagnostic accuracy remains low (60.562.4\%; comparable with FFR 61.7\%), with the best cut-off MLA being $3.4 \mathrm{~mm}^{2}$ across all indices (66). Matsushita et al. also recently reported IVUS-derived MLA to have just 67\% sensitivity and $69 \%$ specificity to predict significant stenosis (iFR $\leq 0.89$ ) requiring a cut-off MLA $<1.8 \mathrm{~mm}^{2}$; equivalent to the FFR $\leq 0.8$ (cut-off MLA $2.0 \mathrm{~mm}^{2}$ ) (67). Together, these data suggest that IVUS provides limited diagnostic accuracy in predicting functionally significant stenosis identified by FFR and iFR.

Reasons for the relatively poor diagnostic accuracy of IVUS in identifying functionally significant CAD is due to several factors including ethnicity, FFR cut-off, vessel size, and high anatomical variations. It is well established that MLA cut-off differs between Asian and Western populations, with comparably lower MLA cut-off values reported in Asian studies of $2.75 v s .3 .0 \mathrm{~mm}^{2}$ (44) and 2.68 vs. $3.03 \mathrm{~mm}^{2}$ (61), respectively. The reasons suggested for this may be associated with a lower body mass index and myocardial mass in Asian patients, but also potentially different lesion characteristics between ethnicity's $(44,61)$. The accuracy of IVUS-derived MLA can be improved when the target FFR is reduced to $<0.75(49,60,61)$. However, most studies adopt an FFR of 0.8 to provide greater clinical relevance and minimise the number of untreated lesions that may result in ischemia, and as such validating an MLA cut-off value for a FFR $<0.75$ may be inappropriate despite the improved accuracy (61). Alternatively, accuracy may be improved by combining other IVUS-derived parameters with the MLA, including stenotic area, minimal luminal diameter (MLD), and lesion length; increasing the sensitivity up to $100 \%$ in one study (37-39). Furthermore, computational "machine learning algorithms" are now being developed which are significantly improving the 
diagnostic accuracy of IVUS to identify lesions with an FFR $\leq 0.8$ up to $86 \%$, with the aim to reduce the need for pressure wire assessment (68).

\section{Basic principles of OCT}

Optical coherence tomography is an intravascular imaging technique which utilises light waves and timedelay information in a modality analogous to ultrasound providing a real-time portrayal of intraluminal and transmural coronary anatomy (69). By analysing the interference pattern of light waves emitted by the catheter using a tool known as the interferometer, the system is able to generate a 'depth-profile' of the luminal walls allowing detailed assessment. Since the light waves used have a much higher frequency than sound waves, the spatial resolution (i.e., the number of pixels used to construct a single image) offered by OCT is greater than that of IVUS, albeit with poorer tissue penetration (70).

First demonstrated in vivo in ophthalmological applications, OCT has found use in the characterisation of intracoronary lesions during percutaneous intervention (71). OCT systems begin by scanning the light beam across a cross-sectional sample to capture an 'A-scan,' which is a 1-dimensional depth profile. As the catheter automatically and rapidly rotates within the portion of coronary artery being assessed, this process is repeated to generate a 2-dimensional 'B-scan' (72). The pullback system then begins, imaging the vessel in this same manner along a pre-determined length of its lumen. The series of B-scans are then compiled into a 3-dimensional, volumetric image known as a 'volume scan' (73). As infrared light cannot penetrate blood, OCT systems require blood to be flushed from the arterial lumen prior to analysis as the presence of erythrocytes causes excess scatter, significantly decreasing imaging quality (71,73). Methods for removing blood from the imaging field are divided into occlusive, involving gentle balloon inflation proximal to the imaged segment, or nonocclusive. Non-occlusive methods are most common with the automated intracoronary administration of crystalloids, iso-osmolar solution, or more commonly contrast (74).

The use of OCT provides several technical and clinical benefits over IVUS. Firstly, OCT systems are capable of achieving faster rotational speeds and hence quicker data acquisition than IVUS, improving workflow and minimising procedural time (75). OCT also provides significantly higher resolution (10-15 versus $100 \mu \mathrm{m})$ when compared with IVUS (76). The improvement in resolution allows the operator to more readily identify dissections, ulceration, thrombus, characterise the size of the coronary vessel and detect stent malapposition (75-77). Furthermore, the features of the backscattered light captured by the catheter on their return can help differentiate atherosclerotic plaques into fibrous or fibrocalcific, and identify presence of a necrotic core, seen as signal-poor regions with poorlydelineated borders and little-to-no backscatter (78). Newer generation OCT catheters also have increased deliverability, being able to be manipulated through tortuous and small calibre coronary vessels.

There are however limitations. Given the light source used by OCT approaches the infrared spectrum with a wavelength average of $1,350 \mathrm{~nm}$, there is an inherent limitation to the depth of field of OCT imaging. This particularly becomes problematic during imaging of the LMCA, where the vessel dimensions can regularly exceed $4 \mathrm{~mm}$. Furthermore, it can be difficult to create a blood-free field in the LMCA due to the high coronary flow volumes in this anatomical location. This can interfere with image acquisition and limits assessment of luminal dimensions and plaque morphology. Finally, OCT cannot be used in the assessment of aorto-ostial lesions of the right or left coronary arteries as it is not possible to adequately clear blood from these locations and thus these lesions lend themselves more towards IVUS assessment (79).

\section{OCT assessment of intermediate lesions}

Similar to IVUS, OCT has also found relevance in the quantification of LMCA lesion severity (Figure 3). Although initial data correlating MLA and FFR was obtained and validated using IVUS (80), these criterion are increasingly being extrapolated to OCT (Figure 4). Experimental phantom models have shown that IVUS may overestimate luminal area by up to $10 \%$, while OCT measurements are highly accurate to 'true' luminal dimensions. Thus, in vivo OCT typically presents smaller cross-sectional luminal areas than IVUS and operators need be aware of this discrepancy when interpreting images. Based on these observations, LMCA lesions with MLA $>5.4 \mathrm{~mm}^{2}$ on OCT are generally considered a safe cut-off for deferral of revascularisation (81).

There is increasing data supporting OCT-derived MLA as a superior predictor of functional ischaemia, when compared with IVUS in non-LMCA. In a study of 186 patients assessing 203 de novo lesions diagnostic efficacy of MLA obtained by OCT and IVUS to predict an FFR of $<0.75$ was assessed. Receiver operator characteristic 


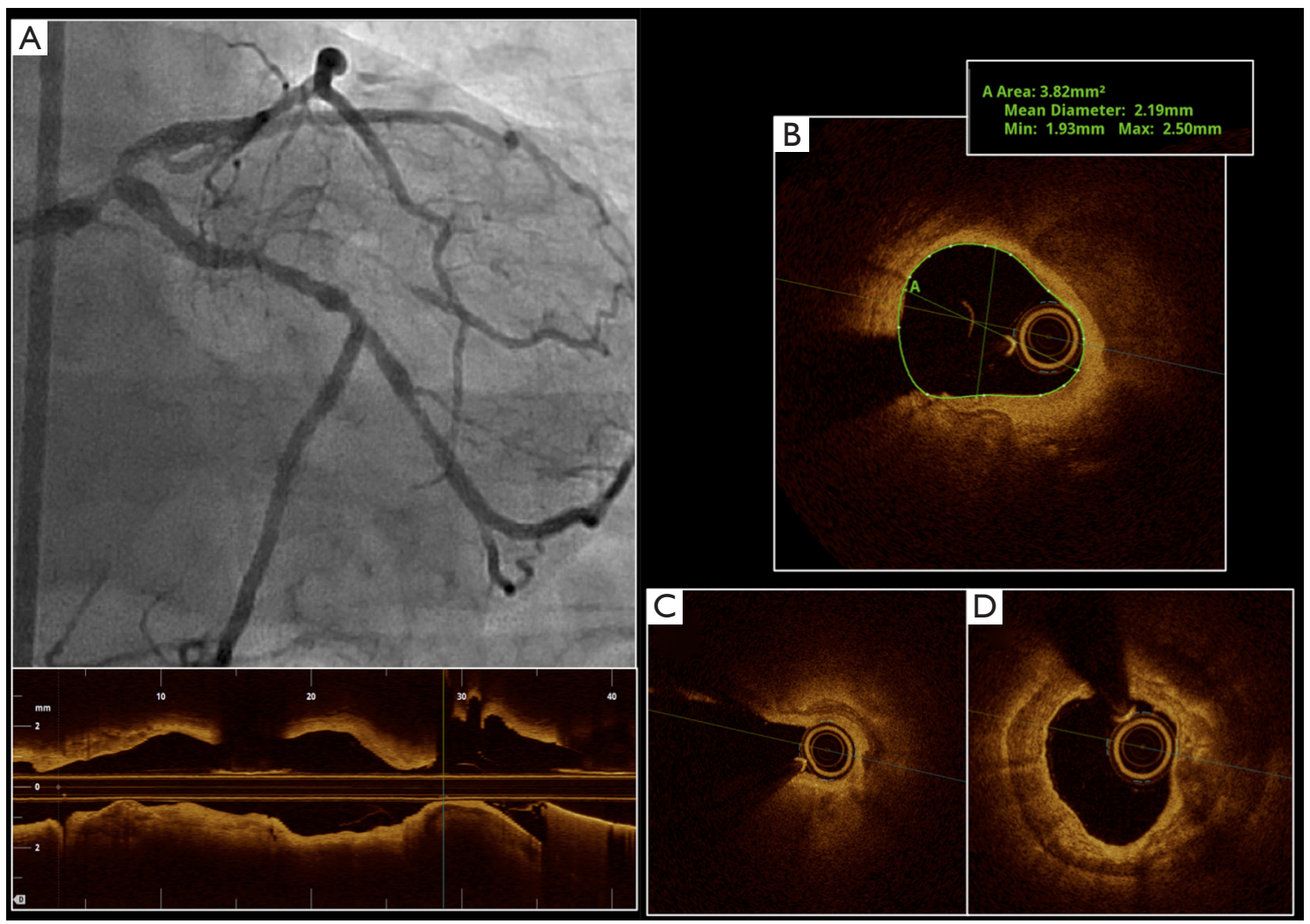

Figure 3 Optical coherence tomography assessment of the distal left main coronary artery bifurcation. Intermediate angiographic distal left main coronary artery (LMCA) lesion (A), with cross sectional optical coherence tomography image (B) of the distal LMCA body with minimal luminal area (MLA) of $3.82 \mathrm{~mm}^{2}$. Importantly, the MLA decreases significantly at the point of confluence (C), which illustrates a slit like orifice to the circumflex artery. Further OCT images of the proximal to mid LAD (D) then highlight concentric calcification, which may act to resist semi-compliant balloon dilatation.

curve analysis showed that OCT-MLA had a statistically significantly better diagnostic efficacy than IVUS-MLA in identifying ischaemia (FFR $<0.75$ ) with the best MLA cutoff values of $1.39 \mathrm{~mm}^{2}$ (AUC: $0.732,95 \%$ CI: $0.660-0.804$ ) and $2.57 \mathrm{~mm}^{2}$ (AUC: 0.615 , 95\% CI: $0.53-0.696$ ) for OCT and IVUS respectively. Interestingly, there was no significant difference between OCT- and IVUS-MLA for the prediction of FFR $\leq 0.80(\mathrm{P}=0.13)$ (82). Although this study suggests that OCT may have superior efficacy to IVUS in detecting functional ischaemia, it must be cautioned that intravascular imaging is currently not interchangeable with FFR in clinical decision making but rather these results suggest that OCT may be preferable to IVUS where there may be doubt, and may reduce the incidence of unnecessary coronary intervention.

Previous meta-analyses support these findings. A metanalysis of 6,919 patients with 7537 non-LMCA lesions found OCT- and IVUS-derived MLA had similar sensitivity in predicting haemodynamically significant lesions (0.732 vs. 0.747, $\mathrm{P}=0.519$ ). OCT-derived MLA however had higher specificity $(76.3 \%$ vs. $66.5 \%, \mathrm{P}<0.001)$ and diagnostic accuracy in detecting flow limiting lesions than IVUS respectively (AUC 0.810 vs. 0.754, $\mathrm{P}=0.045$ ) (83). Of the seven trials included in the analysis specifically utilising OCT, 340 patients and 438 lesions were assessed. These and other similar studies are presented in Table 2. In the largest trial included in the analysis, Reith et al. found that amongst their 100 patients with 142 lesions, receiver operator characteristic analysis demonstrated a moderate diagnostic efficacy of OCT-derived MLA with a best cut-off values of $1.64 \mathrm{~mm}^{2}$ (AUC: $0.836,95 \%$ CI: $0.772-0.901$ ) for the prediction of FFR $\leq 0.80$ (90). These combined results suggest that OCT, with its superior imaging resolution, is the preferred intravascular imaging modality for the 


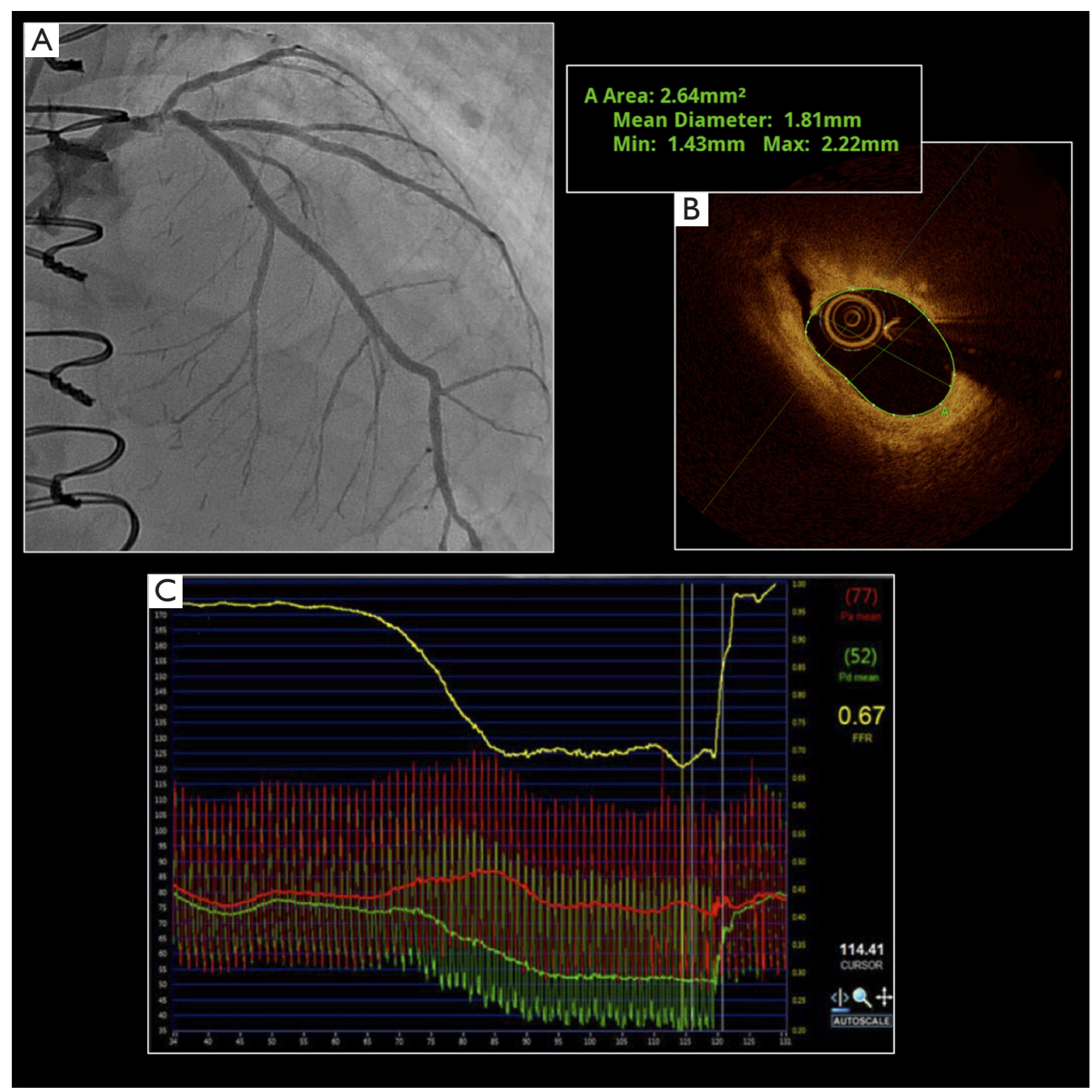

Figure 4 Optical coherence tomography and fractional flow reserve. Intermediate lesion of the distal left main coronary artery (A), which extends into the proximal left anterior descending (LAD). Initial optical coherence tomography images (B) demonstrate a minimal luminal area of $2.64 \mathrm{~mm}^{2}$, with evidence of a small intimal dissection (at 11 o'clock). Fractional flow reserve (FFR) assessment of the vessel (C) demonstrates that the lesion is highly haemodynamically significant (FFR 0.67).

detection of haemodynamically significant stenosis in nonLMCA lesions.

More recent data has also suggested that OCT may be of equal, or even superior, to FFR in guiding PCI decisions in intermediate lesions. The FORZA trial was a direct comparison between OCT and FFR in a 1:1 randomisation pattern. If either FFR or OCT identified a lesion as significant, based on current criteria (FFR $\leq 0.80$ or OCT area stenosis $\geq 75 \%$ or $50-75 \%$ with MLA $<2.5 \mathrm{~mm}^{2}$ ), the patient underwent PCI with optimisation using the same technique. Using pre-defined endpoints of MACE and residual angina, FFR was found to be a more cost-effective strategy with more patients being medically managed
(62.5\% vs. 44.8\%; $\mathrm{P}<0.001)$ however an OCT-guided PCI strategy saw a reduction in all-cause mortality, non-fatal MI, TVR, kidney injury, and significant angina at 13-month follow-up (92). Although the results of this trial are unlikely to alter the current guidelines on physiological assessment of intermediate lesions, it does raise the possibility that an intravascular image-guided approach may yield very similar outcomes and should not be considered inappropriate.

FFR is not without its limitation and as discussed, LMCA lesions may be associated with downstream disease limiting accurate assessment with FFR alone. There is no data utilizing OCT in this clinical scenario however as seen in Figure 2, OCT in combination with FFR assessment 
Table 2 Optimal OCT-defined minimal luminal area (MLA) values to best predict FFR

\begin{tabular}{|c|c|c|c|c|c|c|c|}
\hline Study & Patient/lesions & FFR & Best MLA cut-off (mm²) & Sensitivity (\%) & Specificity (\%) & NPV (\%) & PPV (\%) \\
\hline Usui 2018 (84) & $186 / 203$ & 0.75 & 1.39 & 72.5 & 66.4 & 54.3 & 81.4 \\
\hline Shiono 2012 (85) & $59 / 62$ & 0.75 & 1.91 & 93.5 & 77.4 & 92.3 & 80.6 \\
\hline Belkacemi 2013 (86) & $27 / 27$ & 0.80 & 2.54 & 71.0 & 84.0 & - & - \\
\hline Pawlowski 2013 (88) & $48 / 71$ & 0.80 & 2.05 & 75.0 & 90.0 & 92.6 & 70.6 \\
\hline Zafar 2014 (89) & $30 / 41$ & 0.80 & 1.62 & 70.0 & 97.0 & 91.0 & 89.0 \\
\hline Reith 2015 (90) & $100 / 142$ & 0.80 & 1.64 & 78.8 & 75.8 & 73.4 & 80.8 \\
\hline Burzotta 2018 (91) & $40 / 40$ & 0.80 & 2.50 & 64.0 & 93.0 & - & - \\
\hline
\end{tabular}

NPV, negative predictive value; PPV, positive predictive value; MLA, minimal luminal area; FFR, fractional flow reserve; LMCA, left main coronary artery.

may aid operators in accurate assessment of lesions which require intervention.

\section{Future directions}

Moving forward, the future of OCT and IVUS assessment may lie in coupling with computational fluid dynamics (CFD) simulations of coronary flow and pressure. Use of CFD technology has potential for clinicians to gather simultaneous anatomical and functional assessment of individual coronary lesions. Bezerra et al. (93) have applied these concepts to reconstructed 3D IVUS images, showing a moderate-to-high correlation with invasive pressure wire FFR ( $r=0.79 ; \mathrm{P}<0.001)$. The overall accuracy, sensitivity, specificity, positive predictive value and negative predictive value of this approach was $91 \%, 89 \%, 92 \%, 80 \%$ and $96 \%$, respectively to identify FFR $<0.80$. Similar results have also been shown with OCT, and may be preferable given its higher resolution and more robust estimates of luminal dimensions. In a study of 92 patients with intermediate stenosis undergoing both OCT and FFR assessment, a CFD algorithm was utilised to calculate computational FFR (FFROCT). With a functionally significant stenosis defined as an FFR of $<0.80$, FFROCT resulted in accuracy, sensitivity and specificity of $88 \%, 69 \%$ and $96 \%$ respectively. There remains some hurdles though before this technology is more widely adopted. Firstly, further validation work is required in more diverse lesion and patient cohorts. Secondly, computational time for CFD is still relatively high and this precludes use in a high paced cath lab environment (94). Nevertheless, there is clear potential for the future and there are several industry led collaborations attempting to deliver this technology to clinicians soon.

\section{Conclusions}

Optimal management of intermediate CAD on angiography remains a significant clinical challenge. Although invasive physiological assessment with FFR or iFR remains current gold standard, a number of clinical scenarios may push clinicians towards assessment of lesion severity using intravascular imaging.

\section{Acknowledgments}

Funding: None.

\section{Footnote}

Provenance and Peer Review: This article was commissioned by the Guest Editor (Dennis T. L. Wong) for the series "Intracoronary Imaging" published in Cardiovascular Diagnosis and Therapy. The article was sent for external peer review organized by the Guest Editor and the editorial office.

Conflicts of Interest: The authors have completed the ICMJE uniform disclosure form (available at http://dx.doi. org/10.21037/cdt-20-226). The series "Intracoronary 
Imaging" was commissioned by the editorial office without any funding or sponsorship. ATB reports other from Abbott Vascular, other from Boston Scientific, outside the submitted work. The authors have no other conflicts of interest to declare.

Ethical Statement: The authors are accountable for all aspects of the work in ensuring that questions related to the accuracy or integrity of any part of the work are appropriately investigated and resolved.

Open Access Statement: This is an Open Access article distributed in accordance with the Creative Commons Attribution-NonCommercial-NoDerivs 4.0 International License (CC BY-NC-ND 4.0), which permits the noncommercial replication and distribution of the article with the strict proviso that no changes or edits are made and the original work is properly cited (including links to both the formal publication through the relevant DOI and the license). See: https://creativecommons.org/licenses/by-nc-nd/4.0/.

\section{References}

1. Leape LL, Park RE, Bashore TM, et al. Effect of variability in the interpretation of coronary angiograms on the appropriateness of use of coronary revascularization procedures. Am Heart J 2000;139:106-13.

2. Yong AS, Ng AC, Brieger D, et al. Three-dimensional and two-dimensional quantitative coronary angiography, and their prediction of reduced fractional flow reserve. Eur Heart J 2011;32:345-53.

3. Agostoni P, Biondi-Zoccai G, Van Langenhove G, et al. Comparison of assessment of native coronary arteries by standard versus three-dimensional coronary angiography. Am J Cardiol 2008;102:272-9.

4. Patil CV, Beyar R. Intermediate coronary artery stenosis: evidence-based decisions in interventions to avoid the oculostenotic reflex. Int J Cardiovasc Intervent 2000;3:195-206.

5. Patel MR, Peterson ED, Dai D, et al. Low diagnostic yield of elective coronary angiography. $\mathrm{N}$ Engl J Med 2010;362:886-95.

6. Pijls NH, Sels JW. Functional measurement of coronary stenosis. J Am Coll Cardiol 2012;59:1045-57.

7. De Bruyne B, Pijls NH, Kalesan B, et al. Fractional flow reserve-guided PCI versus medical therapy in stable coronary disease. N Engl J Med 2012;367:991-1001.

8. Tonino PA, De Bruyne B, Pijls NH, et al. Fractional flow reserve versus angiography for guiding percutaneous coronary intervention. N Engl J Med 2009;360:213-24.

9. Gotberg M, Christiansen EH, Gudmundsdottir IJ, et al. Instantaneous Wave-free Ratio versus Fractional Flow Reserve to Guide PCI. N Engl J Med 2017;376:1813-23.

10. Davies JE, Sen S, Dehbi HM, et al. Use of the Instantaneous Wave-free Ratio or Fractional Flow Reserve in PCI. N Engl J Med 2017;376:1824-34.

11. Neumann FJ, Sousa-Uva M, Ahlsson A, et al. [2018 ESC/ EACTS Guidelines on myocardial revascularization]. Kardiol Pol 2018;76:1585-664.

12. Watarai M, Otsuka M, Yazaki K, et al. Applicability of quantitative flow ratio for rapid evaluation of intermediate coronary stenosis: comparison with instantaneous wavefree ratio in clinical practice. Int J Cardiovasc Imaging 2019;35:1963-9.

13. Hwang D, Choi KH, Lee JM, et al. Diagnostic Agreement of Quantitative Flow Ratio With Fractional Flow Reserve and Instantaneous Wave-Free Ratio. J Am Heart Assoc 2019;8:e11605.

14. Botas J. [Assessment and therapeutic guideline of intermediate coronary lesions in the catheterization laboratory]. Rev Esp Cardiol 2003;56:1218-30.

15. Lau JK, Yong AS. Use of fractional flow reserve in different anatomical subsets. Coron Artery Dis 2015;26 Suppl 1:e2-7.

16. Kang SJ, Ahn JM, Song H, et al. Comprehensive intravascular ultrasound assessment of stent area and its impact on restenosis and adverse cardiac events in 403 patients with unprotected left main disease. Circ Cardiovasc Interv 2011;4:562-9.

17. Shammas NW, Radaideh Q, Shammas WJ, et al. The role of precise imaging with intravascular ultrasound in coronary and peripheral interventions. Vasc Health Risk Manag 2019;15:283-90.

18. Erglis A, Jegere S, Narbute I. Intravascular Ultrasoundbased Imaging Modalities for Tissue Characterisation. Interv Cardiol 2014;9:151-5.

19. Brown AJ, Obaid DR, Costopoulos C, et al. Direct Comparison of Virtual-Histology Intravascular Ultrasound and Optical Coherence Tomography Imaging for Identification of Thin-Cap Fibroatheroma. Circ Cardiovasc Imaging 2015;8:e03487.

20. Ma T, Zhou B, Hsiai TK, et al. A Review of Intravascular Ultrasound-based Multimodal Intravascular Imaging: The Synergistic Approach to Characterizing Vulnerable Plaques. Ultrason Imaging 2016;38:314-31.

21. Ahn JM, Kang SJ, Yoon SH, et al. Meta-analysis of 
outcomes after intravascular ultrasound-guided versus angiography-guided drug-eluting stent implantation in 26,503 patients enrolled in three randomized trials and 14 observational studies. Am J Cardiol 2014;113:1338-47.

22. Nerlekar N, Cheshire CJ, Verma KP, et al. Intravascular ultrasound guidance improves clinical outcomes during implantation of both first- and second-generation drug-eluting stents: a meta-analysis. EuroIntervention 2017;12:1632-42.

23. Buccheri S, Franchina G, Romano S, et al. Clinical Outcomes Following Intravascular Imaging-Guided Versus Coronary Angiography-Guided Percutaneous Coronary Intervention With Stent Implantation: A Systematic Review and Bayesian Network Meta-Analysis of 31 Studies and 17,882 Patients. JACC Cardiovasc Interv 2017;10:2488-98.

24. Elgendy IY, Mahmoud AN, Elgendy AY, et al. Intravascular Ultrasound-Guidance Is Associated With Lower Cardiovascular Mortality and Myocardial Infarction for Drug-Eluting Stent Implantation- Insights From an Updated Meta-Analysis of Randomized Trials. Circ J 2019;83:1410-3.

25. Shin DH, Hong SJ, Mintz GS, et al. Effects of Intravascular Ultrasound-Guided Versus AngiographyGuided New-Generation Drug-Eluting Stent Implantation: Meta-Analysis With Individual PatientLevel Data From 2,345 Randomized Patients. JACC Cardiovasc Interv 2016;9:2232-9.

26. Bavishi C, Sardar P, Chatterjee S, et al. Intravascular ultrasound-guided vs angiography-guided drug-eluting stent implantation in complex coronary lesions: Metaanalysis of randomized trials. Am Heart J 2017;185:26-34.

27. Levine GN, Bates ER, Blankenship JC, et al. 2011 ACCF/AHA/SCAI Guideline for Percutaneous Coronary Intervention. A report of the American College of Cardiology Foundation/American Heart Association Task Force on Practice Guidelines and the Society for Cardiovascular Angiography and Interventions. J Am Coll Cardiol 2011;58:e44-122.

28. Neumann FJ, Sousa-Uva M, Ahlsson A, et al. 2018 ESC/ EACTS Guidelines on myocardial revascularization. Eur Heart J 2019;40:87-165.

29. Lindstaedt M, Spiecker M, Perings C, et al. How good are experienced interventional cardiologists at predicting the functional significance of intermediate or equivocal left main coronary artery stenoses? Int J Cardiol 2007;120:254-61.

30. Cameron A, Kemp HG, Jr., Fisher LD, et al. Left main coronary artery stenosis: angiographic determination. Circulation 1983;68:484-9.

31. Abizaid AS, Mintz GS, Abizaid A, et al. One-year follow-up after intravascular ultrasound assessment of moderate left main coronary artery disease in patients with ambiguous angiograms. J Am Coll Cardiol 1999;34:707-15.

32. Jasti V, Ivan E, Yalamanchili V, et al. Correlations between fractional flow reserve and intravascular ultrasound in patients with an ambiguous left main coronary artery stenosis. Circulation 2004;110:2831-6.

33. Fassa AA, Wagatsuma K, Higano ST, et al. Intravascular ultrasound-guided treatment for angiographically indeterminate left main coronary artery disease: a longterm follow-up study. J Am Coll Cardiol 2005;45:204-11.

34. de la Torre Hernandez JM, Hernandez Hernandez F, Alfonso F, et al. Prospective application of pre-defined intravascular ultrasound criteria for assessment of intermediate left main coronary artery lesions results from the multicenter LITRO study. J Am Coll Cardiol 2011;58:351-8.

35. Kang SJ, Lee JY, Ahn JM, et al. Intravascular ultrasoundderived predictors for fractional flow reserve in intermediate left main disease. JACC Cardiovasc Interv 2011;4:1168-74.

36. Park SJ, Ahn JM, Kang SJ, et al. Intravascular ultrasoundderived minimal lumen area criteria for functionally significant left main coronary artery stenosis. JACC Cardiovasc Interv 2014;7:868-74.

37. Takagi A, Tsurumi Y, Ishii Y, et al. Clinical potential of intravascular ultrasound for physiological assessment of coronary stenosis: relationship between quantitative ultrasound tomography and pressure-derived fractional flow reserve. Circulation 1999;100:250-5.

38. Lee CH, Tai BC, Soon CY, et al. New set of intravascular ultrasound-derived anatomic criteria for defining functionally significant stenoses in small coronary arteries (results from Intravascular Ultrasound Diagnostic Evaluation of Atherosclerosis in Singapore [IDEAS] study). Am J Cardiol 2010;105:1378-84.

39. Briguori C, Anzuini A, Airoldi F, et al. Intravascular ultrasound criteria for the assessment of the functional significance of intermediate coronary artery stenoses and comparison with fractional flow reserve. Am J Cardiol 2001;87:136-41.

40. Koo BK, Yang HM, Doh JH, et al. Optimal intravascular ultrasound criteria and their accuracy for defining the functional significance of intermediate coronary 
stenoses of different locations. JACC Cardiovasc Interv 2011;4:803-11.

41. Ben-Dor I, Torguson R, Deksissa T, et al. Intravascular ultrasound lumen area parameters for assessment of physiological ischemia by fractional flow reserve in intermediate coronary artery stenosis. Cardiovasc Revasc Med 2012;13:177-82.

42. Kang SJ, Ahn JM, Song H, et al. Usefulness of minimal luminal coronary area determined by intravascular ultrasound to predict functional significance in stable and unstable angina pectoris. Am J Cardiol 2012;109:947-53.

43. Yang HM, Tahk SJ, Lim HS, et al. Relationship between intravascular ultrasound parameters and fractional flow reserve in intermediate coronary artery stenosis of left anterior descending artery: intravascular ultrasound volumetric analysis. Catheter Cardiovasc Interv 2014;83:386-94.

44. Han JK, Koo BK, Park KW, et al. Optimal intravascular ultrasound criteria for defining the functional significance of intermediate coronary stenosis: an international multicenter study. Cardiology 2014;127:256-62.

45. Waksman R, Legutko J, Singh J, et al. FIRST: Fractional Flow Reserve and Intravascular Ultrasound Relationship Study. J Am Coll Cardiol 2013;61:917-23.

46. Cui M, Zhu D, Guo LJ, et al. Usefulness of lumen area parameters determined by intravascular ultrasound to predict functional significance of intermediate coronary artery stenosis. Chin Med J (Engl) 2013;126:1606-11.

47. Naganuma T, Latib A, Costopoulos C, et al. The role of intravascular ultrasound and quantitative angiography in the functional assessment of intermediate coronary lesions: correlation with fractional flow reserve. Cardiovasc Revasc Med 2014;15:3-7.

48. Gonzalo N, Escaned J, Alfonso F, et al. Morphometric assessment of coronary stenosis relevance with optical coherence tomography: a comparison with fractional flow reserve and intravascular ultrasound. J Am Coll Cardiol 2012;59:1080-9.

49. Ben-Dor I, Torguson R, Gaglia MA, Jr., et al. Correlation between fractional flow reserve and intravascular ultrasound lumen area in intermediate coronary artery stenosis. EuroIntervention 2011;7:225-33.

50. Brown AJ, Giblett JP, Bennett MR, et al. Anatomical plaque and vessel characteristics are associated with hemodynamic indices including fractional flow reserve and coronary flow reserve: A prospective exploratory intravascular ultrasound analysis. Int J Cardiol 2017;248:92-6.
51. Ye Y, Yang M, Zhang S, et al. Percutaneous coronary intervention in left main coronary artery disease with or without intravascular ultrasound: A meta-analysis. PLoS One 2017;12:e0179756.

52. Wang Y, Mintz GS, Gu Z, et al. Meta-analysis and systematic review of intravascular ultrasound versus angiography-guided drug eluting stent implantation in left main coronary disease in 4592 patients. BMC Cardiovasc Disord 2018;18:115.

53. Liu XM, Yang ZM, Liu XK, et al. Intravascular ultrasoundguided drug-eluting stent implantation for patients with unprotected left main coronary artery lesions: A singlecenter randomized trial. Anatol J Cardiol 2019;21:83-90.

54. de la Torre Hernandez JM, Baz Alonso JA, Gomez Hospital JA, et al. Clinical impact of intravascular ultrasound guidance in drug-eluting stent implantation for unprotected left main coronary disease: pooled analysis at the patient-level of 4 registries. JACC Cardiovasc Interv 2014;7:244-54.

55. Oviedo C, Maehara A, Mintz GS, et al. Intravascular ultrasound classification of plaque distribution in left main coronary artery bifurcations: where is the plaque really located? Circ Cardiovasc Interv 2010;3:105-12.

56. Fearon WF, Yong AS, Lenders G, et al. The impact of downstream coronary stenosis on fractional flow reserve assessment of intermediate left main coronary artery disease: human validation. JACC Cardiovasc Interv 2015;8:398-403.

57. McDaniel MC, Eshtehardi P, Sawaya FJ, et al. Contemporary clinical applications of coronary intravascular ultrasound. JACC Cardiovasc Interv 2011;4:1155-67.

58. Park SJ, Kang SJ, Ahn JM, et al. Visual-functional mismatch between coronary angiography and fractional flow reserve. JACC Cardiovasc Interv 2012;5:1029-36.

59. Costa MA, Sabate M, Staico R, et al. Anatomical and physiologic assessments in patients with small coronary artery disease: final results of the Physiologic and Anatomical Evaluation Prior to and After Stent Implantation in Small Coronary Vessels (PHANTOM) trial. Am Heart J 2007;153:296.e1-7.

60. Nascimento BR, de Sousa MR, Koo BK, et al. Diagnostic accuracy of intravascular ultrasound-derived minimal lumen area compared with fractional flow reserve--metaanalysis: pooled accuracy of IVUS luminal area versus FFR. Catheter Cardiovasc Interv 2014;84:377-85.

61. Jang JS, Shin HC, Bae JS, et al. Diagnostic Performance of Intravascular Ultrasound-Derived Minimal Lumen 
Area to Predict Functionally Significant Non-Left Main Coronary Artery Disease: a Meta-Analysis. Korean Circ J 2016;46:622-31.

62. Koh JS, Koo BK, Kim JH, et al. Relationship between fractional flow reserve and angiographic and intravascular ultrasound parameters in ostial lesions: major epicardial vessel versus side branch ostial lesions. JACC Cardiovasc Interv 2012;5:409-15.

63. de la Torre Hernandez JM, Lopez-Palop R, Garcia Camarero $\mathrm{T}$, et al. Clinical outcomes after intravascular ultrasound and fractional flow reserve assessment of intermediate coronary lesions. Propensity score matching of large cohorts from two institutions with a differential approach. EuroIntervention 2013;9:824-30.

64. Nam CW, Yoon HJ, Cho YK, et al. Outcomes of percutaneous coronary intervention in intermediate coronary artery disease: fractional flow reserve-guided versus intravascular ultrasound-guided. JACC Cardiovasc Interv 2010;3:812-7.

65. Park KW, Kang SH, Yang HM, et al. Impact of intravascular ultrasound guidance in routine percutaneous coronary intervention for conventional lesions: data from the EXCELLENT trial. Int J Cardiol 2013;167:721-6.

66. Lee JM, Choi KH, Koo BK, et al. Intravascular ultrasound or optical coherence tomography-defined anatomic severity and hemodynamic severity assessed by coronary physiologic indices. Rev Esp Cardiol (Engl Ed) 2019.

67. Matsushita K, Hibi K, Okada K, et al. Comparison between instantaneous wave-free ratio versus morphometric assessments by intracoronary imaging. Heart Vessels 2019;34:926-35.

68. Lee JG, Ko J, Hae H, et al. Intravascular ultrasound-based machine learning for predicting fractional flow reserve in intermediate coronary artery lesions. Atherosclerosis 2020;292:171-7.

69. Mintz GS. Clinical utility of intravascular imaging and physiology in coronary artery disease. J Am Coll Cardiol 2014;64:207-22.

70. Johnson PM, Patel J, Yeung M, et al. Intra-coronary imaging modalities. Curr Treat Options Cardiovasc Med 2014;16:304.

71. Bezerra HG, Costa MA, Guagliumi G, et al. Intracoronary optical coherence tomography: a comprehensive review clinical and research applications. JACC Cardiovasc Interv 2009;2:1035-46.

72. Ha FJ, Giblett JP, Nerlekar N, et al. Optical Coherence Tomography Guided Percutaneous Coronary Intervention. Heart Lung Circ 2017;26:1267-76.
73. Prati F, Jenkins MW, Di Giorgio A, et al. Intracoronary optical coherence tomography, basic theory and image acquisition techniques. Int J Cardiovasc Imaging 2011;27:251-8.

74. Tanigawa J, Barlis P, Di Mario C. Intravascular optical coherence tomography: optimisation of image acquisition and quantitative assessment of stent strut apposition. EuroIntervention 2007;3:128-36.

75. Maehara A, Matsumura M, Ali ZA, et al. IVUS-Guided Versus OCT-Guided Coronary Stent Implantation: A Critical Appraisal. JACC Cardiovasc Imaging 2017;10:1487-503.

76. Ali ZA, Karimi Galougahi K, Maehara A, et al. Intracoronary Optical Coherence Tomography 2018: Current Status and Future Directions. JACC Cardiovasc Interv 2017;10:2473-87.

77. Takarada S, Imanishi T, Liu Y, et al. Advantage of next-generation frequency-domain optical coherence tomography compared with conventional time-domain system in the assessment of coronary lesion. Catheter Cardiovasc Interv 2010;75:202-6.

78. Tearney GJ, Regar E, Akasaka T, et al. Consensus standards for acquisition, measurement, and reporting of intravascular optical coherence tomography studies: a report from the International Working Group for Intravascular Optical Coherence Tomography Standardization and Validation. J Am Coll Cardiol 2012;59:1058-72.

79. Fujino Y, Attizzani GF, Bezerra HG, et al. Serial assessment of vessel interactions after drug-eluting stent implantation in unprotected distal left main coronary artery disease using frequency-domain optical coherence tomography. JACC Cardiovasc Interv 2013;6:1035-45.

80. Ahn JM, Kang SJ, Mintz GS, et al. Validation of minimal luminal area measured by intravascular ultrasound for assessment of functionally significant coronary stenosis comparison with myocardial perfusion imaging. JACC Cardiovasc Interv 2011;4:665-71.

81. Jegere S, Narbute I, Erglis A. Use of intravascular imaging in managing coronary artery disease. World J Cardiol 2014;6:393-404.

82. Usui E, Yonetsu T, Kanaji Y, et al. Efficacy of Optical Coherence Tomography-derived Morphometric Assessment in Predicting the Physiological Significance of Coronary Stenosis: Head-to-Head Comparison with Intravascular Ultrasound. EuroIntervention 2018;13:e2210-8.

83. Ramasamy A, Chen Y, Zanchin T, et al. Optical 
coherence tomography enables more accurate detection of functionally significant intermediate non-left main coronary artery stenoses than intravascular ultrasound: A meta-analysis of 6919 patients and 7537 lesions. Int J Cardiol 2020;301:226-34.

84. Usui E, Yonetsu T, Kanaji Y, et al. Relationship between optical coherence tomography-derived morphological criteria and functional relevance as determined by fractional flow reserve. J Cardiol 2018;71:359-66.

85. Shiono Y, Kitabata H, Kubo T, et al. Optical coherence tomography-derived anatomical criteria for functionally significant coronary stenosis assessed by fractional flow reserve. Circ J 2012;76:2218-25.

86. Belkacemi A, Stella PR, Ali DS, et al. Diagnostic accuracy of optical coherence tomography parameters in predicting in-stent hemodynamic severe coronary lesions: validation against fractional flow reserve. Int J Cardiol 2013;168:4209-13.

87. Pyxaras SA, Tu S, Barbato E, et al. Quantitative angiography and optical coherence tomography for the functional assessment of nonobstructive coronary stenoses: comparison with fractional flow reserve. Am Heart J 2013;166:1010-8.e1.

88. Pawlowski T, Prati F, Kulawik T, et al. Optical coherence tomography criteria for defining functional severity of intermediate lesions: a comparative study with FFR. Int J Cardiovasc Imaging 2013;29:1685-91.

89. Zafar H, Ullah I, Dinneen K, et al. Evaluation of

Cite this article as: Nogic J, Prosser H, O'Brien J, Thakur U, Soon K, Proimos G, Brown AJ. The assessment of intermediate coronary lesions using intracoronary imaging. Cardiovasc Diagn Ther 2020;10(5):1445-1460. doi: 10.21037/cdt-20-226 hemodynamically severe coronary stenosis as determined by fractional flow reserve with frequency domain optical coherence tomography measured anatomical parameters. J Cardiol 2014;64:19-24.

90. Reith S, Battermann S, Hellmich M, et al. Correlation between optical coherence tomography-derived intraluminal parameters and fractional flow reserve measurements in intermediate grade coronary lesions: a comparison between diabetic and non-diabetic patients. Clin Res Cardiol 2015;104:59-70.

91. Burzotta F, Nerla R, Hill J, et al. Correlation between frequency-domain optical coherence tomography and fractional flow reserve in angiographically-intermediate coronary lesions. Int J Cardiol 2018;253:55-60.

92. Burzotta F, Leone AM, Aurigemma C, et al. Fractional Flow Reserve or Optical Coherence Tomography to Guide Management of Angiographically Intermediate Coronary Stenosis: A Single-Center Trial. JACC Cardiovasc Interv 2020;13:49-58.

93. Bezerra CG, Hideo-Kajita A, Bulant CA, et al. Coronary fractional flow reserve derived from intravascular ultrasound imaging: Validation of a new computational method of fusion between anatomy and physiology. Catheter Cardiovasc Interv 2019;93:266-74.

94. Kern MJ, Seto AH. Fractional flow reserve from intravascular ultrasound imaging: Computational fluid dynamics to the rescue? Catheter Cardiovasc Interv 2019;93:275-7. 